\title{
McKittrick-Wheelock syndrome: a rare cause of acute renal failure and hypokalemia not to be overlooked
}

\section{Manuel Alfredo Podestà, David Cucchiari, Elisa Merizzoli, Ugo Elmore, Claudio Angelini \& Salvatore Badalamenti}

To cite this article: Manuel Alfredo Podestà, David Cucchiari, Elisa Merizzoli, Ugo Elmore, Claudio Angelini \& Salvatore Badalamenti (2014) McKittrick-Wheelock syndrome: a rare cause of acute renal failure and hypokalemia not to be overlooked, Renal Failure, 36:5, 811-813, DOI: 10.3109/0886022X.2014.890056

To link to this article: https://doi.org/10.3109/0886022X.2014.890056

\section{Published online: 24 Feb 2014.}

Submit your article to this journal $\pi$
III Article views: 582

Q View related articles ¿

View Crossmark data $[\pi$

Citing articles: 5 View citing articles $\sqsubset$ 


\title{
McKittrick-Wheelock syndrome: a rare cause of acute renal failure and hypokalemia not to be overlooked
}

\author{
Manuel Alfredo Podestà ${ }^{1}$, David Cucchiari ${ }^{1}$, Elisa Merizzoli ${ }^{1}$, Ugo Elmore ${ }^{2}$, Claudio Angelini ${ }^{1}$, and \\ Salvatore Badalamenti ${ }^{1}$ \\ ${ }^{1}$ Nephrology and Dialysis Unit, Humanitas Clinical and Research Center, Rozzano, Milan, Italy and ${ }^{2}$ Minimally Invasive Surgery Unit, \\ Humanitas Clinical and Research Center, Rozzano, Milan, Italy
}

\begin{abstract}
McKittrick-Wheelock syndrome is a rare disorder in which a colorectal tumor (usually a villous adenoma) determines secretory mucous diarrhea, which in turn leads to prerenal acute renal failure, hyponatremia, hypokalemia and metabolic acidosis. Even though the outcome is usually favorable with complete recovery after surgery, the diagnosis is often delayed, making the patient susceptible to life-threatening complications, mainly severe acidosis and hypokalemia. We present two paradigmatic cases with extreme electrolytes imbalance and complete recovery following the appropriate treatment. The pathogenesis of this degenerative condition is discussed in detail.
\end{abstract}

\section{Keywords}

Acute renal failure, hypokalemia, hyponatremia, metabolic acidosis, villous adenoma

\section{History}

Received 21 October 2013

Revised 8 January 2014

Accepted 26 January 2014

Published online 24 February 2014

\section{Background}

McKittrick-Wheelock syndrome is a rare disorder, with approximately 50 cases reported in international literature since its first description in $1954 .{ }^{1-3}$ This syndrome is defined by a triad that includes: (1) secretory mucous diarrhea determined by a benign/malignant colorectal tumor, which leads to (2) a profound volume and salt depletion resulting in hyponatremia and prerenal acute renal failure (ARF) and (3) hypokalemia with metabolic acidosis. ${ }^{4,5}$ Even though the outcome is usually favorable with complete recovery after surgery, the diagnosis is often delayed, making the patient susceptible to life-threatening complications, mainly severe acidosis and hypokalemia.

\section{Case 1}

A 72-year-old woman was referred to our unit for hypokalemia. She described mucous diarrhea and intermittent colic hypogastric pain, whose onset was referred to be 5 months prior to presentation. She also reported one week of symptoms worsening and urine output reduction. Her medical history included mild hypertension, total thyroidectomy and hepatitis C infection successfully treated with interferon. The patient's medications included olmesartan and hydrochlorothiazide. She denied diabetes mellitus, theophylline or

Address correspondence to Manuel Alfredo Podestà, via Manzoni 56, 20089, Rozzano, Milan, Italy. Tel: +00393420036285; E-mail: manpodes@gmail.com laxatives use, recent diaphoresis or vomit, and she reported a normal diet.

Physical examination showed mild disorientation, dry skin and mucous membranes, low blood pressure $(100 / 70 \mathrm{mmHg})$, tachycardia (115 bpm) and tachypnea (24 breaths/min). Her abdomen was tender and diffusely painful on palpation, bowel sounds were heard in all quadrants. The patient did not demonstrate guarding and Murphy's and McBurney's signs were negative. Digital rectal examination revealed a firm mass with an irregular surface. Soft and watery stool was present with no visible blood. An ECG was obtained, showing flattened $\mathrm{T}$ waves, $\mathrm{U}$ waves and wide PR interval. Laboratory investigations demonstrated acute kidney injury (AKI) with a previously known normal renal function (baseline creatinine $0.7 \mathrm{mg} / \mathrm{dL}$ ), severe hypokalemia and hyponatremia, metabolic acidosis (Table 1). Urinary sediment and dipstick were unremarkable.

Normal saline $(2.000 \mathrm{~mL} /$ day $)$ and continuous potassium chloride infusion $(80 \mathrm{mEq} /$ day $)$ were started, with rapid recovery of kidney function (creatinine $0.9 \mathrm{mg} / \mathrm{dL}$ at day 5) and resolution of hyponatremia. Serum potassium concentration remained equal to $3.0 \mathrm{mEq} / \mathrm{L}$ at $72 \mathrm{~h}$ despite continuous infusion. Flexible sigmoidoscopy and CT scan revealed a circumferential villous mass extending upward from the rectum. Magnetic resonance imaging of the pelvis confirmed the presence of the pathological lesion extended throughout the rectum, occupying the lumen of the bowel $\left(360^{\circ}\right)$ and a suspected infiltration of the perirectal fat (Figure 1). Colonoscopy confirmed the presence of a circumferential villous mass extending from the dentate line by $15 \mathrm{~cm}$. 
Table 1. Patients' baseline features.

\begin{tabular}{lcc}
\hline & Patient 1 & Patient 2 \\
\hline Creatinine (mg/dL) & 11.7 & 4.8 \\
Urea (mg/dL) & 227 & 180 \\
Sodium (mEq/L) & 122 & 124 \\
Potassium (mEq/L) & 2.9 & 2.4 \\
Chloride (mEq/L) & 96 & 94 \\
Urinary potassium (mEq/day) & 17 & 20 \\
Blood pH & 7.25 & 7.18 \\
Bicarbonate (mmol/L) & 13.6 & 12.4 \\
pCO $(\mathrm{mmHg})$ & 31 & 33 \\
Anion gap & 12.4 & 15.6 \\
\hline
\end{tabular}

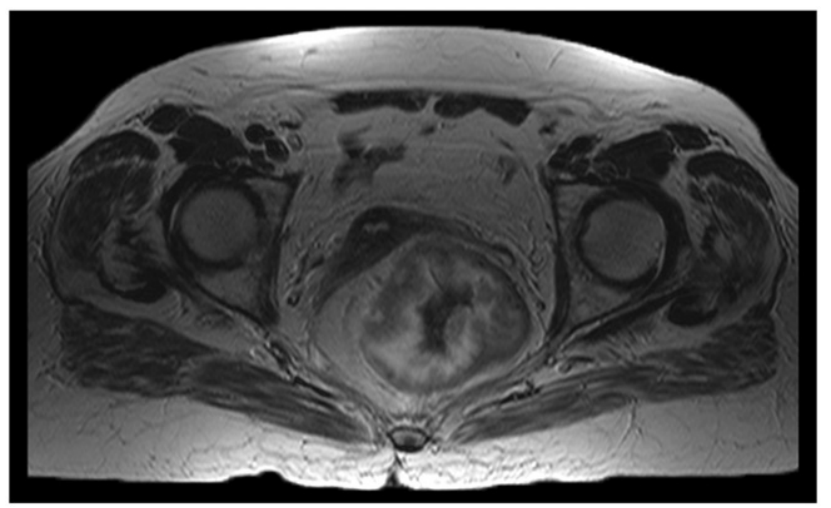

Figure 1. Magnetic resonance imaging showing the giant villous adenoma of the colon in patient 1 .

Endoscopic ultrasound confirmed the thickening of the mucosa and submucosa with no signs of infiltration of the muscularis and serosa and sparing of internal and external anal sphincters.

On the sixth day from admission, the patient underwent a laparoscopic anterior resection of the rectum with colo-anal anastomosis and terminal ileostomy. The postoperative course was uneventful. The histology confirmed a sessile villous adenoma with high-grade dysplasia without evidence of cancer. Two days after surgery, serum potassium concentration returned to the normal range $(3.6 \mathrm{mEq} / \mathrm{L})$.

\section{Case 2}

An 83-year-old man presented to the Emergency Department complaining of fatigue and light-headedness, associated to a sudden decrease in urinary output. His medical history included diuretic-treated hypertension, alcoholic cirrhosis and atrio-ventricular block type III that required pacemaker (PM) implantation. The patient was also previously diagnosed a villous adenoma of the rectum with low-grade dysplasia that was endoscopically unresectable and was left untreated because he denied the consent to surgical resection.

On physical examination he appeared to be dehydrated with blood pressure of $95 / 60 \mathrm{mmHg}$ and heart rate of 105 bpm. Heart, abdomen and lung examination were unremarkable. A digital rectal examination was performed, revealing a soft irregular mass on the posterior surface of the rectum. Mucous stool was present with no visible blood. Blood chemistry results were consistent with AKI, hypokalemia, hyponatremia and metabolic acidosis (Table 1).

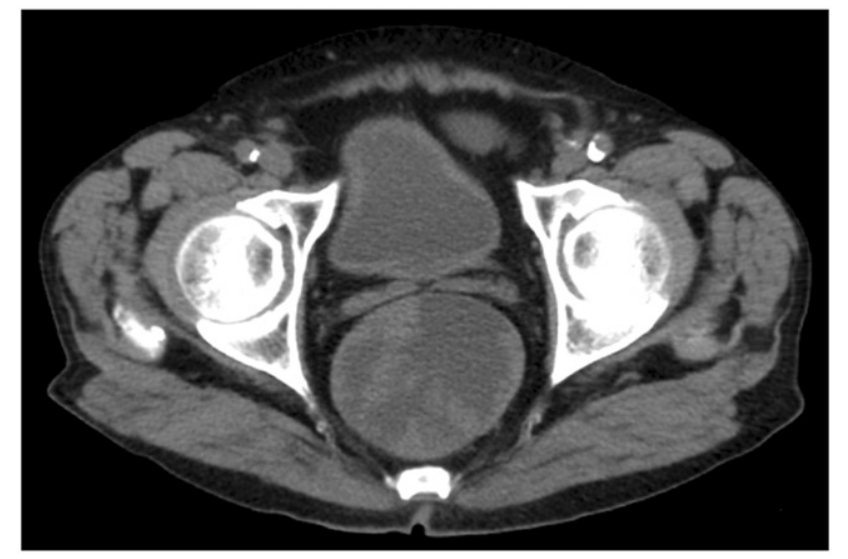

Figure 2. Computed tomography showing the giant villous adenoma of the colon in patient 2 .

Normal saline $(2.000 \mathrm{~mL} /$ day $)$ was rapidly administered along with potassium infusion $(40 \mathrm{mEq} /$ day $)$, potassium oral supplementation ( $48 \mathrm{mEq} /$ day) and spironolactone. A rapid recovery of renal function was obtained (creatinine $0.9 \mathrm{mg} / \mathrm{dL}$ at day 7) along with correction of hyponatremia ( $\mathrm{Na}+$ $137 \mathrm{mEq} / \mathrm{L}$ at day 7). With this management, serum potassium concentration kept improving, with normalization at discharge, two weeks after admission ( $\mathrm{K}+3.5 \mathrm{mEq} / \mathrm{L})$. Following these findings, colonoscopy was performed, showing the known villous adenoma, which measured $15 \mathrm{~cm}$ in its maximum diameter. The polypoid lesion was bleeding and occupying the lumen of the bowel $\left(360^{\circ}\right)$. CT abdomen scan showed no signs of local infiltration (Figure 2).

The patient underwent transanal excision with combined mininvasive approach. The postoperative course was uneventful. The histology confirmed a sessile villous adenoma with high-grade dysplasia. For the persistence of residual tissue in the rectal ampulla, after one year the patient underwent the completion of the endoscopic resection.

\section{Discussion}

McKittrick-Wheelock syndrome is usually associated with villous adenomas. These tumors show secretory activity in approximately $3 \%$ of cases, but in order to determine the aforementioned symptoms, they need to be large enough and to be localized in the distal part of the colon. ${ }^{6}$ In general, the more distal and the larger the adenoma is, the more likely fluids and electrolytes excreted cannot be reabsorbed. In some cases, when losses are compensated by fluid and salts intake, diarrhea can persist for years before the patient is forced to seek medical advice. When the tumor secretion exceeds increased intake and renal compensatory mechanisms, symptoms become evident.

The cause of hypersecretion, often exceeding $10 \mathrm{~mL} / \mathrm{kg} /$ day (a fact that has led some authors to refer to this syndrome as to "Neoplastic Cholera'), ${ }^{7}$ is still debated. Enterocytes have a bicarbonate-exchanger on apical surface, which could be inappropriately activated in these cells, leading to depletion of bicarbonate, sodium and potassium. It has been suggested that prostaglandin E2 is involved as a locally-released secretagogue, ${ }^{8}$ thus justifying the use of 
cyclo-oxygenase irreversible inhibitors such as indomethacin in order to limit further losses. ${ }^{9}$

Fluid losses can be as high as $3 \mathrm{~L} /$ day, depending on the size and localization of the adenoma. Metabolic acidosis is due to stool loss of bicarbonate, a common occurrence in case of secretory diarrhea. Acidosis tends to mask the real degree of hypokalemia because it inhibits $\mathrm{Na}+\mathrm{K}+$ pumps, redistributing potassium from the intracellular to the extracellular compartment. Therefore, close attention should be paid to potassium depletion, which is frequently worse than predicted by serum concentration. Anion gap is influenced by both chloride secretion and organic acids retention, with the latter increasing when AKI develops. However, the presence of an altered water and sodium balance makes anion gap unreliable. ${ }^{10}$ Hyponatremia ensues on the basis of sodium loss and extracellular fluid volume depletion, which triggers nonosmotic release of vasopressin (ADH), resulting in dilutional hyponatremia. Finally, extreme dehydration leads to prerenal AKI, which is reversible if fluid losses are promptly corrected.

Aggressive hydration is usually enough to correct both hyponatremia and prerenal azotemia. Acidosis does not require specific correction with bicarbonate infusion, unless it is severe $(\mathrm{pH}<7.10)$. Oral and intravenous potassium supplementation, on the other hand, is often not enough to correct and maintain an adequate potassium serum concentration. Moreover, correction of AKI and acidosis can worse serum potassium concentration, making fluid administration challenging. Definitive correction of this disorder is achieved only by surgical means: since endoscopic resection is frequently difficult, laparoscopic resection is usually considered the treatment of choice.

In conclusion, McKittrick-Wheelock syndrome is a rare disorder associated to a villous adenoma causing secretory diarrhea, electrolyte imbalances and prerenal AKI. Owing to its rarity, this syndrome is either frequently overlooked or it is suspected only in later stages of disease. This delay in the diagnosis can lead to life-threatening complications, thus findings of hypokalemia due to extrarenal potassium loss, associated to diarrhea and AKI should prompt an appropriate workup to rule out this rare syndrome.

\section{Declaration of interest}

The authors have no conflict of interest or financial ties to disclose.

\section{References}

1. McKittrick LS, Wheelock FC. Carcinoma of the colon. 1954. Dis Colon Rectum. 1997;40(12):1494-1495; discussion 1495-1496.

2. Martins HS, Brandao-Neto RA, de Carvalho AL, et al. McKittrickWheelock syndrome: a cause of severe hydro-electrolyte disorders in ED. Am J Emerg Med. 2007;25(9):1083 e1081-e1083.

3. Lepur D, Klinar I, Mise B, et al. McKittrick-Wheelock syndrome: a rare cause of diarrhoea. Eur J Gastroenterol Hepatol. 2006; 18(5):557-559.

4. Lee YS, Lin HJ, Chen KT. McKittrick-Wheelock syndrome: a rare cause of life-threatening electrolyte disturbances and volume depletion. J Emerg Med. 2012;43(3):e171-e173.

5. Pucci G, Rondelli F, Avenia N, et al. Acute renal failure and metabolic alkalosis in a patient with colorectal villous adenoma (McKittrick-Wheelock syndrome). Surgery. 2013;154(3):643-644.

6. Choi WH, Ryuk J, Kim HJ, et al. A case of giant rectal villous tumor with severe fluid-electrolyte imbalance treated by laparoscopic low anterior resection. J Korean Surg Soc. 2012; 82(5):325-329.

7. Winstanley V, Little MA, Wadsworth C, et al. The McKittrickWheelock syndrome: a case of acute renal failure due to neoplastic cholera. Ren Fail. 2008;30(4):469-473.

8. Jacob H, Schlondorff D, St Onge G, et al. Villous adenoma depletion syndrome. Evidence for a cyclic nucleotide-mediated diarrhea. Dig Dis Sci. 1985;30(7):637-641.

9. Smelt AH, Meinders AE, Hoekman K, et al. Secretory diarrhea in villous adenoma of rectum: effect of treatment with somatostatin and indomethacin. Prostaglandins. 1992;43(6):567-572.

10. Feldman M, Soni NJ, Dickson B. Use of sodium concentration and anion gap to improve correlation between serum chloride and bicarbonate concentrations. J Clin Lab Anal. 2006;20(4):154-159. 